\title{
A Nonparametric Approach to Model Error Modeling
}

\author{
Anders Stenman, Fredrik Tjärnström \\ Department of Electrical Engineering \\ Linköpings universitet, SE-581 83 Linköping, SWEDEN \\ URL: http://www. control.isy.liu.se \\ Email: stenman@isy.liu.se, fredrikt@isy.liu.se
}

March 16, 2000

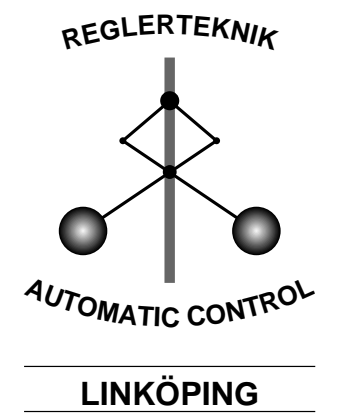

Report no.: LiTH-ISY-R-2230

To be presented at the IFAC Symposium on System Identification, Santa Barbara, California, USA, June 21-23, 2000.

Technical reports from the Automatic Control group in Linköping are available by anonymous ftp at the address ftp.control.isy.liu.se. This report is contained in the PDF file 2230.pdf. 


\title{
A NONPARAMETRIC APPROACH TO MODEL ERROR MODELING
}

\author{
Anders Stenman ${ }^{*, 1}$ Fredrik Tjärnström* \\ * Department of Electrical Engineering, Linköpings universitet, \\ SE-581 83 Linköping, SWEDEN. Fax: +4613282622
}

\begin{abstract}
To validate an estimated model and evaluate its reliability is an important part of the system identification process. Recent work on model validation has shown that the use of explicit model error models provide a better way of visualizing the possible deficiencies of the nominal model. Previous contributions have mainly focused on parametric black-box models for estimating the error model. However, this requires that a correct model order for the error model has to be selected. Here we suggest an adaptive and nonparametric frequency-domain method that estimates the frequency response of the model error by an automatic procedure. A benefit with this approach is that the tuning can be done locally, i.e., that different resolutions can be used in different frequency bands. The ideas are based on local polynomial regression and utilize a statistical criterion for selecting the optimal resolution.
\end{abstract}

Keywords: model error modeling, non-parametric regression, frequency-response methods

\section{INTRODUCTION}

The concept of model validation has received an increased attention in recent years. Approaches with unknown but bounded disturbances (UBB), stochastic embedding, control oriented model validation, $\mathcal{H}_{\infty}$-identification etc, typically have the ambition of providing more reliable models for control design. Another approach in this direction is the concept of model error modeling (Ljung, 1999a), where parametric black-box models have been utilized with the intention to reveal the model error's properties more in detail. However, this requires that the parameterization and the order of the error model are correctly selected.

In this contribution we suggest a nonparametric frequency-domain approach that estimates the model error's frequency response by an automatic and adaptive procedure. The method is based on local polynomial modeling ideas and is an appli-

\footnotetext{
1 Corresponding author. Email: stenman@isy.liu.se
}

cation of the procedure proposed in Stenman et al. (1999). It has the advantage that the estimation can be tuned locally for each frequency, i.e., that different resolutions can be used in different frequency bands. Moreover, it also provides an easy way of getting pointwise confidence bands for the estimate. This can be a very useful tool when visualizing the properties of the model error.

The organization of the paper is as follows. Section 2 gives the general setup. Section 3 shows how the concept of local polynomial models can be used for providing more detailed information about the model error. Section 4 illustrates the concept with a numerical example, and in Section 5, finally, some concluding remarks are drawn.

\section{PRELIMINARIES}

The setup for model validation (or model invalidation) can be described as follows: We assume that we have at hand a nominal input-output model $\hat{G}(q)$ along with a validation data set, 


$$
Z^{N} \triangleq\{(u(1), y(1)), \ldots,(u(N), y(N))\},
$$

where $y(t)$ and $u(t)$ represent the output and the input of the system. We would now like to construct a testing procedure by which we may falsify or invalidate the given model. That is, devise a procedure that makes us able to say that it is unlikely (or even impossible) that the model has generated the validation data. If the model is not falsified by this test, however, it seems reasonable to say that the model has passed the validation.

A common theme among a majority of the traditional approaches for model validation, is the idea of evaluating the model's quality by testing its performance on fresh data. This has typically involved a whiteness test on the residuals,

$$
\varepsilon(t) \triangleq y(t)-\hat{G}(q) u(t), \quad t=1, \ldots, N,
$$

(or an examination of the correlation between the residuals and past inputs) in combination with a qualitative comparison of the true and predicted outputs. In the next section, though, we shall see how more accurate information can be obtained by proper modeling of the model error.

\section{DIRECT MODEL ERROR MODELING}

An alternative to the above statistical approaches for validation, is to try to estimate the parts in $\varepsilon(t)$ that depend on unmodeled dynamics with blackbox models. This was referred to as model error modeling by Ljung (1999a). The idea was however first introduced in Ljung et al. (1991).

The most general error model is perhaps the nonlinear model

$$
\varepsilon(t)=m_{\Delta}\left(\varepsilon^{t-1}, u^{t}\right)+w(t),
$$

where $m_{\Delta}(\cdot)$ is an arbritrary nonlinear mapping that takes care of the unmodeled dynamics, and $w(t)$ is a disturbance. However, to keep things simple, it appears to be reasonable to specialize into linear models of the form

$$
\varepsilon(t)=\Delta(q) u(t)+w(t) .
$$

\subsection{Modeling Approaches}

An obvious way of modeling $\Delta(q)$ in (4) is of course to utilize high order FIR or ARX models. This was the approach adopted by Ljung (1999a). The problem is then of course how to choose the right model structure and model order. If these were known this approach would be impossible to beat since its variance is then known to meet the Cramèr-Rao bound.

Another possibility is to use the standard nonparametric frequency domain methods, based on the Blackman-Tukey method (Blackman and Tukey, 1958). The idea is to compute an estimate of the model error's frequency response $\Delta\left(e^{i \omega}\right)$ by smoothing the ratio

$$
\hat{\hat{\Delta}}\left(e^{i \omega_{k}}\right) \triangleq \frac{\Xi\left(\omega_{k}\right)}{U\left(\omega_{k}\right)}=\frac{\sum_{t=1}^{N} \varepsilon(t) e^{-i \omega_{k} t}}{\sum_{t=1}^{N} u(t) e^{-i \omega_{k} t}} .
$$

The quantity (5) is normally referred to as the empirical transfer function estimate, ETFE, of the error model. This is a very "raw" and noisy estimate of the frequency response, and typically needs to be further refined. The classical solution is to average over neighboring ETFE values using a fixed window $W_{\gamma}(\cdot)$ (Ljung, 1999b);

$$
\hat{\Delta}\left(e^{i \omega_{k}}\right)=\frac{\int_{-\pi}^{\pi} W_{\gamma}(\omega-\xi)\left|U_{N}(\xi)\right|^{2} \hat{\hat{\Delta}}\left(e^{i \xi}\right) d \xi}{\int_{-\pi}^{\pi} W_{\gamma}(\omega-\xi)\left|U_{N}(\xi)\right|^{2} d \xi} .
$$

One can show that the variance of $\hat{\Delta}\left(e^{i \omega_{k}}\right)$ then approaches

$$
\operatorname{Var} \hat{\Delta}\left(e^{i \omega_{k}}\right) \approx \frac{0.75 \gamma}{N} \cdot \frac{\Phi_{v}(\omega)}{\Phi_{u}(\omega)},
$$

provided the standard Hamming window is used. The main drawback here is that one usually needs a high $\gamma$ to get a good frequency resolution. This will typically give a very noisy estimate (Stenman et al., 1999). A remedy is to use the nonparametric frequency-domain approach that was introduced in Stenman et al. (1999). A short description of this procedure will be given next.

\subsection{A Local Polynomial Approach}

The basic idea behind the local polynomial approach is to replace the averaging (6) with a more general smoothing procedure. The frequency function of the model error, $\Delta\left(e^{i \omega}\right)$, is assumed to be smooth and is estimated by fitting a $p$ th degree polynomial to the data located within a sliding window. That is, for each frequency $\omega$, an estimate is obtained via the weighted regression problem; minimize

$$
\begin{array}{r}
V(\omega, \beta)=\sum_{k=1}^{N}\left|\hat{\hat{\Delta}}\left(e^{i \omega_{k}}\right)-\sum_{j=0}^{p} \beta_{j}\left(\omega_{k}-\omega\right)^{j}\right|^{2} \\
\times w_{k}(\omega) \cdot \frac{1}{\sigma_{k}^{2}},
\end{array}
$$

subject to $\beta_{j} \in \mathbb{C}$, where the $w_{k}(\omega)$ 's are the window weights. If $\hat{\beta}_{j}, j=0, \ldots, p$, denote the minimizers of (7), an estimate of the model error frequency response is given by

$$
\hat{\Delta}\left(e^{i \omega}\right)=\hat{\beta}_{0} .
$$

The window weights are usually chosen according to

$$
w_{k}(\omega)=K\left(\frac{\omega_{k}-\omega}{h}\right),
$$


where $h$ is a smoothness parameter (usually referred to as the bandwidth) controlling the spread of the window, and $K(\cdot)$ is a kernel function with support on $[-1,1]$ assigning weights to each remote frequency-domain measurement according to its distance from $\omega$. However, it has been shown that it is also possible to select the weights via an explicit optimization stage that aims at minimizing the mean square error of (8) subject to the weights. Consult Stenman (1999) for details around this.

We have in (7) also included the inverse variance of the measurements as an extra weighting. It can be estimated either directly from the frequency domain data (5) using standard variance estimation techniques for nonparametric models (Loader, 1999), or by utilizing theoretical formulas (Guillaume et al., 1996).

3.2.1. Local Model Tuning It is well known that the resolution parameter $h$ has a critical impact on the resulting estimate since it governs a trade-off between bias and variance. Methods that use the available data to produce a bandwidth are usually referred to as bandwidth selectors, and have been extensively studied within the statistical literature, see, for instance, Fan and Gijbels (1996).

As in previous applications we here consider an adaptive, localized and generalized $C_{p}$ approach with variance penalty $\alpha$ (Cleveland and Loader, 1994)

$$
\begin{gathered}
C(\omega, h) \triangleq \sum_{k=1}^{N} \frac{w_{k}(\omega)}{\sigma_{k}^{2} \operatorname{tr}(\mathbf{W})}\left|\hat{\hat{\Delta}}\left(e^{i \omega_{k}}\right)-\tilde{\Delta}\left(e^{i \omega_{k}}\right)\right|^{2} \\
-1+\alpha \frac{\operatorname{tr}\left(\left(\boldsymbol{\Omega}^{T} \mathbf{W} \mathbf{V} \boldsymbol{\Omega}\right)^{-1}\left(\boldsymbol{\Omega}^{T} \mathbf{W}^{2} \mathbf{V} \boldsymbol{\Omega}\right)\right)}{\operatorname{tr}(\mathbf{W})}
\end{gathered}
$$

Here $\boldsymbol{\Omega}$ is a Vandermonde-type matrix containing the basis functions for the polynomial model, and $\mathbf{W}$ and $\mathbf{V}$ are diagonal matrices containing the weights $w_{k}(\omega)$ and the inverse variances $1 / \hat{\sigma}_{k}^{2}$, respectively. The parameter $\alpha$ is an extra tuning knob that provides the possibility of changing the penalty on the variance term in (10). It defaults to 2 , but can be increased if the variability of estimates gets too high (see Stenman (1999)). Thus, for each frequency $\omega$, a local choice of resolution $h=h(\omega)$ can be obtained by minimizing $C(\omega, h)$ subject to $h$.

3.2.2. Confidence Bands for the Estimate The local polynomial smoothing method provides point estimates of the frequency function at specified frequencies. However, in the context of model error modeling, it is in general of more interest to assess the uncertainty associated with the error model rather than the model itself. (The esti- mated frequency response of the model error can in fact be quite bad as a consequence of the low signal-to-noise ratio of the residuals).

Nevertheless, it turns out that computing pointwise confidence bands is a rather straightforward thing to do. Note that the frequency response via (7) effectively is estimated by means of a linear smoothing operation,

$$
\hat{\Delta}\left(e^{i \omega}\right)=\sum_{k=1}^{N} W_{k}(\omega) \hat{\hat{\Delta}}\left(e^{i \omega_{k}}\right),
$$

where the linear weights $\left\{W_{k}(\omega)\right\}$ are determined either explicitly by the optimization approach, or implicitly by a kernel and a polynomial fitting procedure. Hence it follows that the variance of (11) is approximately

$$
\operatorname{Var} \hat{\Delta}\left(e^{i \omega}\right)=\hat{\sigma}^{2}(\omega) \cdot\|\mathbf{w}(\omega)\|^{2},
$$

where $\hat{\sigma}^{2}(\omega)$ denotes an estimate of the complex noise variance at the frequency $\omega$, and

$$
\mathbf{w}(\omega) \triangleq\left(W_{1}(\omega) \ldots W_{N}(\omega)\right)^{T}
$$

is the vector of smoothing weights. It is thus straightforward to construct approximate confidence bands for $\Delta\left(e^{i \omega}\right)$ (or more precisely, $\left.\mathrm{E} \hat{\Delta}\left(e^{i \omega}\right)\right)$.

It can be shown that the real and imaginary parts of $\hat{\Delta}\left(e^{i \omega}\right)$ are asymptotically uncorrelated and jointly normally distributed with variances equal to half the value of (12). See, e.g., Ljung (1999b). Using Gauss' approximation formula we find that the absolute value of the smoothed estimate is asymptotically normally distributed with variance

$$
\operatorname{Var}\left|\hat{\Delta}\left(e^{i \omega}\right)\right| \approx \frac{\hat{\sigma}^{2}(\omega) \cdot\|\mathbf{w}(\omega)\|^{2}}{2}
$$

(Ljung, 1999b, Exercise 9G.1). From this, confidence bands along the amplitude curve can be constructed. A confidence band of degree $95 \%$ at each frequency will be formed as

$$
\left|\hat{\Delta}\left(e^{i \omega}\right)\right| \pm \frac{1.96}{\sqrt{2}} \cdot \hat{\sigma}(\omega) \cdot\|\mathbf{w}(\omega)\| .
$$

Remember, however, that these values are only approximations. To obtain more accurate confidence bands, a more detailed analysis of the distributions of the amplitude and phase must be performed and some bias adjustment might be required (see, e.g., Fan and Gijbels (1996)). Different possibilities also exist depending on which quantities we want to display (the amplitude, the logarithm of the amplitude and so on). It might also be possible to utilize numerical methods such as point-mass approaches or bootstrapping (Efron and Tibshirani, 1993).

\section{AN EXAMPLE}

Let us illustrate the above model error modeling approach with an example (adapted from Ljung 
(1999a)). A data set of length 2000 was generated using the "system"

$$
y(t)=\frac{B(q)}{F(q)} u(t)+v(t)
$$

with

$$
\begin{aligned}
B(q)= & q^{-1}+0.5 q^{-2} \\
F(q)= & 1-2.2 q^{-1}+2.42 q^{-2} \\
& -1.87 q^{-3}+0.7225 q^{-4}
\end{aligned}
$$

where the input $u(t)$ was chosen as a zero mean Gaussian sequence with unit variance, and the disturbance $v(t)$ was an additive Gaussian noise sequence with zero mean and variance 0.5 . The data set was divided into two equally sized parts, one for estimation and one for validation. A second order output-error model $\hat{G}(q)$ was identified using the estimation dataset and the system identification toolbox in MATLAB (Ljung, 1997). This model will be referred to as the nominal model in the remainder of the section.

Figure 1 shows the frequency response of the nominal model (dashed curve) along with its $95 \%$ confidence bands (shaded). The frequency response of the true system (14) has also been included (solid line). It is clear that the confidence bands do not contain the true system. This is consistent
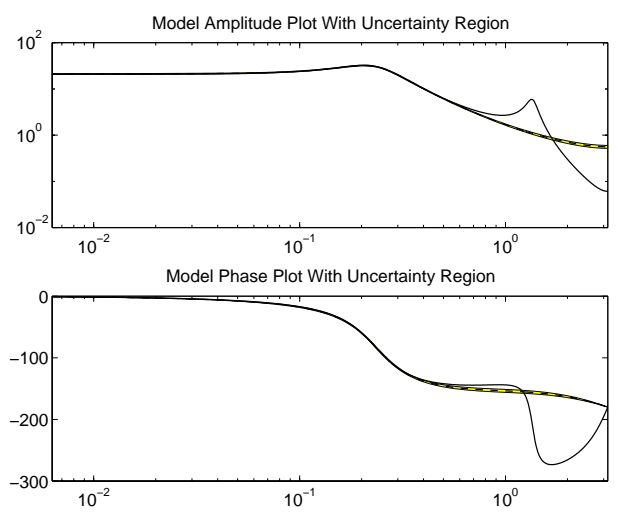

Fig. 1. The frequency response of the nominal model (dashed), the true system (solid) and its $95 \%$ confidence bands (shaded).

with Figure 2, where the correlation function of the residuals and the cross-correlation between residuals and past inputs are displayed. We see that the values of the cross-correlation function do not seem to pass a traditional-type validation test. The conclusion is therefore that there are some significant errors present in the nominal model. It is however not obvious what types of errors they represent.

Let us see what conclusions we can draw by using the procedure outlined in Section 3.2. Figure 3 shows the amplitude curve of the model error model (solid line) along with its uncertainty regions (shaded). For comparison, the amplitude
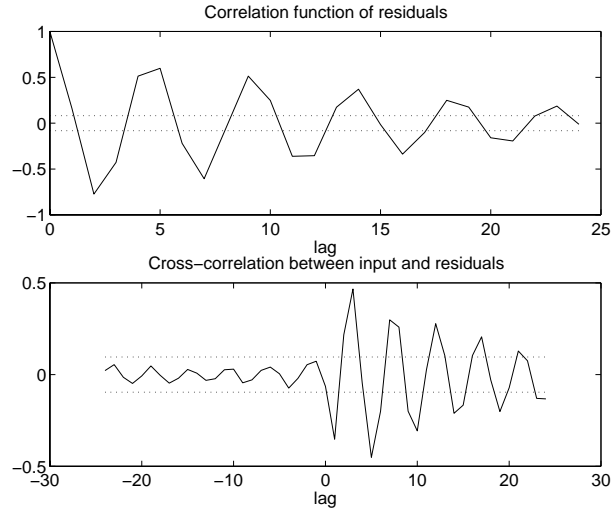

Fig. 2. Correlation and cross-correlation functions with $95 \%$ confidence bands.

curve of the "true" model error has also been included (solid line). The uncertainty regions tell us that there are significant model errors in the frequency range above $0.2 \mathrm{rad} / \mathrm{s}$. The frequency
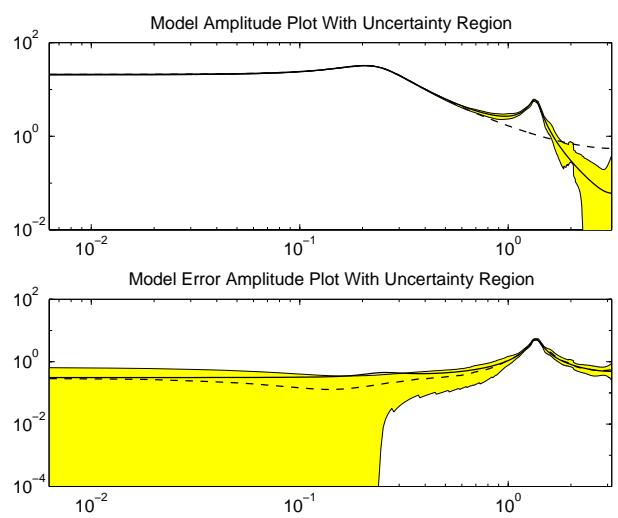

Fig. 3. Lower plot: The amplitude curve of the estimated model error (dashed) using local smoothing methods along with its uncertainty bands (shaded). The solid curve is the true model error. Upper plot: The amplitude curve of the nominal model (dashed) and of the true system (solid) together with the uncertainty region obtained as the sum of the nominal model and the model error model uncertainty (shaded).

response has been estimated and tuned locally according to (7) and (10), using a local quadratic model structure $(p=2)$, the tricube kernel

$$
K(u)= \begin{cases}\left(1-|u|^{3}\right)^{3}, & |u| \leq 1, \\ 0, & |u|>1,\end{cases}
$$

and a variance estimate $\hat{\sigma}_{k}^{2}$ obtained as

$$
\hat{\sigma}_{k}=\frac{\hat{\Phi}_{v}\left(\omega_{k}\right)}{\left|U_{N}\left(\omega_{k}\right)\right|^{2}},
$$

where $U_{N}\left(\omega_{k}\right)$ is the discrete Fourier transform of $u(t)$ and $\hat{\Phi}_{v}\left(\omega_{k}\right)$ is an initial estimate of the noise spectrum. For more details see Stenman et al. (1999). The selected bandwidths are displayed in Figure 4. It is clearly seen that the bandwidth 


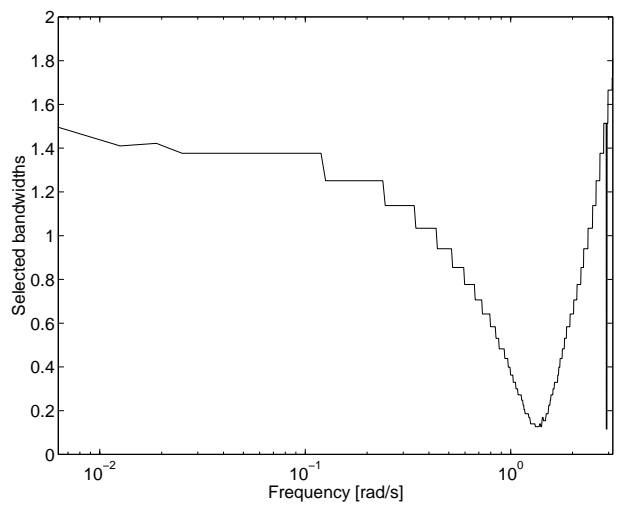

Fig. 4. The selected bandwidths according to the $C_{p}$ criterion, here $\alpha=5$ was used.

selector (10) adapts the resolution to the properties of the data.

We compare this result with what is obtained using parametric and standard frequency domain methods.

In the nonparametric case we use $\gamma=100$ to make sure that no resonance peaks are missed. The result can be found in Figure 5. We see that we find the resonance peak quite accurately, but the noisiness makes it difficult to accurately interpret the type of model errors present in the nominal model. The "size" of the confidence regions using these nonparametric methods are more or less the same. This is to be expected since the use the same a priori information.
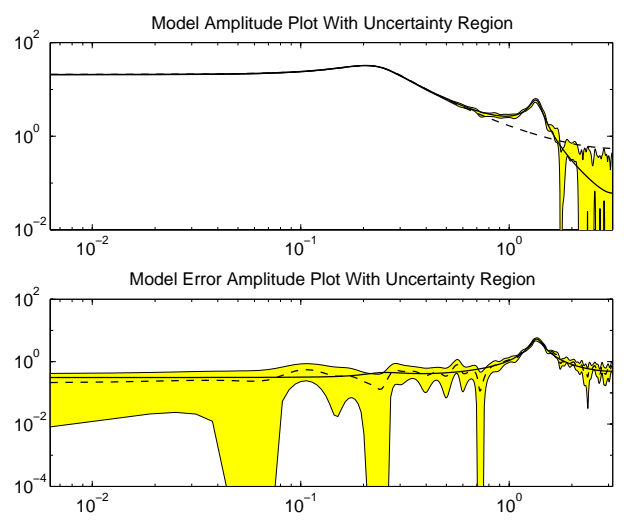

Fig. 5. Lower plot: The amplitude curve of the estimated model error (dashed) using traditional nonparametric frequency domain methods with $\gamma=100$ along with its uncertainty bands (shaded). The solid curve is the true model error. Upper plot: The amplitude curve of the nominal model (dashed) and of the true system (solid) together with the uncertainty region obtained as the sum of the nominal model and the model error model uncertainty (shaded).

When using parametric models, the problem is always to decide the structure and order of the model. Here we have used an ARX model of order 15, see Figure 6. We used an ARX model to eliminate the risk of ending up in local minima. The order was chosen more at random, but high enough to pass a standard validation test. The regions are a little bit smaller than those obtained with the other methods. Note that the ARX structure is not correct in this example, the correct structure is actually an OE model of order 6. This implies that there is some bias in the estimate, which in turn implies that the variance estimate of the parametric model might not be correct. Therefore one can argue that the local smoothing methods presented here is a better alternative since its parameter free and produce very good estimates, i.e., has high resolution and high smoothing at the same time.
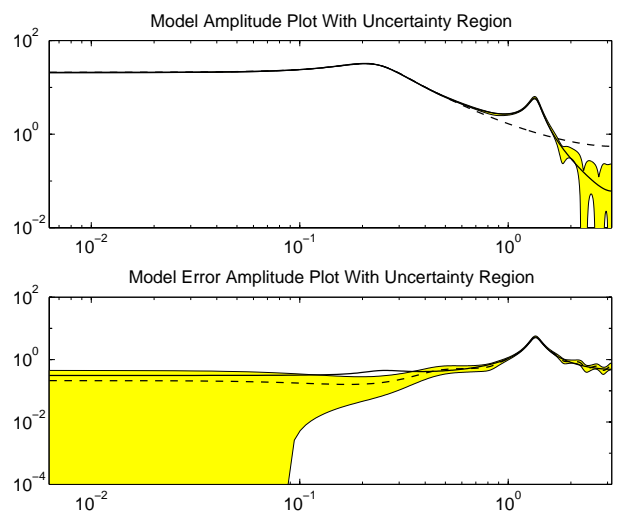

Fig. 6. Lower plot: The amplitude curve of the estimated model error (solid) using an ARX model of order 15 along with its uncertainty bands (shaded). The solid curve is the true model error. Upper plot: The amplitude curve of the nominal model (dashed) and of the true system (solid) together with the uncertainty region obtained as the sum of the nominal model and the model error model uncertainty (shaded).

\section{CONCLUSIONS}

To validate an estimated model and evaluate its reliability is an important part of the system identification process. We have demonstrated that the use of explicit model error models is a convenient way of visualizing the possible deficiencies of the nominal model, and provides a useful tool for the validation procedure.

Based on local polynomial modeling and a statistical bandwidth selection scheme, we have proposed a method that estimates the model error's frequency response using an adaptive and automatic procedure. This allows for a frequency-dependent choice of frequency resolution and gives several 
advantages over traditional parametric and nonparametric approaches. The frequency response of the error model typically exhibit fine details to different degrees in different frequency bands. The results are more easily interpreted than standard frequency domain methods and have variance properties similar to parametric models. Our adaptive approach thus gives a useful alternative compared to other multi-resolution techniques like, e.g., wavelets.

\section{REFERENCES}

Blackman, R.B. and J.W. Tukey (1958). The Measurement of Power Spectra. Dover, New York.

Cleveland, W.S. and C. Loader (1994). Smoothing by local regression: Principles and methods. Technical report. AT\&T Bell Laboratories. 600 Mountain Avenue, Murray Hill, NJ 07974, USA.

Efron, B. and R. J. Tibshirani (1993). An Introduction to the Bootstrap. Chapman \& Hall.

Fan, J. and I. Gijbels (1996). Local Polynomial Modelling and Its Applications. Chapman \& Hall.

Guillaume, P., I. Kollár and R. Pintelon (1996). Statistical analysis of nonparametric transfer function estimates. IEEE Transactions on Instrumentation and Measurement 45(2), 594600.

Ljung, L. (1997). System Identification ToolboxUser's Guide. The Mathworks Inc. 24 Prime Park Way, Natick, MA.

Ljung, L. (1999a). Model validation and model error modeling. In: The Aström Symposium on Control (B. Wittenmark and A. Rantzer, Eds.). Studentlitteratur, Lund, Sweden.

Ljung, L. (1999b). System Identification: Theory for the User. 2nd ed.. Prentice-Hall.

Ljung, L., B. Wahlberg and H. Hjalmarsson (1991). Model quality: The roles of prior knowledge and data information. In: Proceedings of the 30th IEEE Conference on Decision and Control. Brighton, U.K.. pp. 273-278.

Loader, C. (1999). Local Regression and Likelihood. Springer, New York.

Stenman, A. (1999). Model on Demand: Algorithms, Analysis and Applications. PhD thesis. Dept of EE, Linköping University. SE-581 83, Linköping, Sweden.

Stenman, A., F. Gustafsson, D.E. Rivera, L. Ljung and T. McKelvey (1999). On adaptive smoothing of empirical transfer function estimates. In: Preprints of the 14th World Congress of IFAC, Beijing, P.R. China (H.F. Chen and B. Wahlberg, Eds.). Vol. H. Elsevier Science. pp. 415-420. 